\title{
Rigid Body Dynamics using Clifford Algebra
}

\author{
J.M. Selig and E. Bayro-Corrochano
}

\begin{abstract}
In this paper the dynamics of rigid bodies is recast into a Clifford algebra formalism. Specifically, the algebra $C \ell(0,6,2)$, is used and it is shown how velocities, momenta and inertias can be represented by elements of this algebra. The equations of motion for a rigid body are simply derived by differentiating the momentum of the body.
\end{abstract}

Mathematics Subject Classification (2000). Primary 70E15; Secondary 11E88.

Keywords. Rigid bodies, Dynamics, Clifford algebra.

\section{Introduction}

In this work we present a Clifford algebra for representing the dynamics of rigid bodies. Much of the motivation for doing this comes from robotics. In robotics we commonly have several rigid bodies connected by simple joints. The equations of motion of serial robots, where the bodies are joined to each other in a serial chain, are quite simple to derive. Much of this simplicity comes from two ideas usually absent from the physics literature on mechanics. Rather, two ideas which simplify the analysis of single rigid bodies actually complicate matters when we have several bodies to consider. The first idea is to split the translational and rotational motion. In robotics things are a lot simpler if we treat translational and rotational velocities on an equal footing. The second usual simplification that we must abandon is the body-fixed co-ordinate frame, if we have several rigid bodies in which one should we fix the co-ordinate frame? So we choose a standard fixed inertial frame to work in. This means however, that the inertia operators for the rigid bodies will not be constant but will change as the bodies move with respect to our standard frame.

A simple method of finding the equations of motion for robots in this way is Screw theory. In Screw theory the rotational and translational velocities of a rigid body are combined to form a single six-dimensional vector called a screw. In essence, these screws are elements of the Lie algebra to the group of rigid body 
motions, and modern approaches to Screw theory use this Lie theoretic point of view to much advantage.

By using Clifford algebra we get a common algebraic framework in which graphic, vision, kinematics and now dynamics problems can be expressed. As these functions of robots become more closely integrated in practical systems we expect that a uniform way of analysing these features will become useful.

Another area which may benefit from this work is so called "Game Physics", see [8] for example. The problem here is to simulate systems of rigid bodies for computer games. Many different approaches have been suggested recently, in particular the use of techniques from linear programming, see [16]. These complimentarity problems would seem to be well suited to formulation in a Clifford algebra. There may be some gain in overall efficiency if the graphics system used to display the results was also based on Clifford algebra.

It has been suggested elsewhere that Clifford algebras could form the basis of efficient computer algorithms, see [2] for instance. Certainly, modern processor architectures seem to favour algebraic methods. Much of the development of computer hardware at present is driven by the need to speed up graphics. The computations required in graphics are in essence, computations in a Clifford algebra. Indeed the use of quaternions to represent rotations is commonplace in commercial computer graphics systems, see [17]. So there is some hope that the support for Clifford algebra in future hardware may be better than it is now and hence the advantage of Clifford algebras will increase.

However, to claim that the results presented here will lead to fast algorithms for dynamics is premature. The algebra we use is rather large and it is well known that this will lead to large memory requirements and slow algorithms.

There have been previous attempts to write rigid body dynamics using Clifford algebra. Hestenes [5] presents an essentially 3 dimensional approach using $C \ell(3)$. Linear velocities are represented as vectors and angular velocities as bivectors. So linear and angular velocities can be combined into a single term in the algebra. Forces and torques are treated in much the same way. The power can then be found by taking the Clifford product of the velocities and forces and extracting the scalar part. This neat formulation has some drawbacks however. The action of the group of rigid body motions on the velocities and forces is a little awkward, rotations are effected by conjugation with unit elements of even grade but translation must be added as vectors. As mentioned above the velocities or screws form a Lie algebra with a Lie bracket operation, this operation is not modelled in the Clifford algebra. Neither is the coadjoint action of a Lie algebra element on an element in the dual of the Lie algebra. This operation is especially important in dynamics as it gives the so called Coriolis terms in the equations of motion. Finally, the inertia matrix is represented as an operation that maps bivectors to bivectors. It is not an element of the Clifford algebra.

Hestenes [6] and Hestenes and Fasse [7] introduce another method using the algebra $C \ell(4,1)$. In this algebra linear velocities are represented by elements of grade 2 and angular velocities by grade 4 elements. The action of the rigid body 
motion group on these screws is now much cleaner, both rotations and translations are represented by conjugations with certain unit elements of even degree. However, the action of the group on the force/torque elements is not clear and many of the other drawbacks mentioned above still apply. Abou El Dahab [1] gave a formulation of Hamiltonian mechanics using Clifford algebra. In some respects the approach taken is similar to the approach taken in this work. Configuration space is assumed to be the space of vectors in a Clifford algebra and phase space is constructed by enlarging the algebra to include the conjugate momentum vectors. The symplectic geometry of phase space is realised using a distinguished bivector.

The original intention of this work was simply to apply Hestenes' $C \ell(4,1)$ approach to the dynamics of robots. However, the drawbacks mentioned above meant that this could not be done as explicitly as was required. This failure prompted the search for an alternative approach.

This alternative approach, reported below, uses the algebra $C \ell(0,6,2)$. This arises from the duplication of the algebra $C \ell(0,3,1)$. The algebra $C \ell(0,3,1)$ contains a copy of the group of rigid body motions $S E(3)$ as well as its Lie algebra, the screws, and elements corresponding to the points, lines and planes of Euclidean 3-space. By duplicating the algebra, elements can be found to represent the momenta, dual to velocities and the inertia which transforms velocities into momenta. Because the algebra we are using is degenerate, we have to introduce the shuffle product. We also find it helpful to use the distinguished involution on the algebra which exchanges elements from one copy of $C \ell(0,3,1)$ with their corresponding elements in the other copy.

We begin with a brief review of rigid body dynamics using the standard Screw formalism.

\section{Rigid Body Dynamics}

The neatest formalism for rigid body dynamics uses 6-dimensional vectors to represent velocities and momenta. These vector are effectively elements of the Lie algebra of the group of rigid body motions, $S E(3)$. In partitioned form these vectors can be written,

$$
\mathbf{s}=\left(\begin{array}{l}
\boldsymbol{\omega} \\
\mathbf{v}
\end{array}\right)=\left(\begin{array}{l}
\omega_{x} \\
\omega_{y} \\
\omega_{z} \\
v_{x} \\
v_{y} \\
v_{z}
\end{array}\right)
$$

Instantaneously the body is rotating about an axis with angular velocity $\boldsymbol{\omega}$ and simultaneously translating along the same axis with linear velocity $\mathbf{v}$. These $6-$ dimensional velocity vectors are called twists, or sometimes screws. 
Similarly momenta can be written as,

$$
\mathcal{P}=\left(\begin{array}{l}
\mathbf{l} \\
\mathbf{p}
\end{array}\right)=\left(\begin{array}{c}
l_{x} \\
l_{y} \\
l_{z} \\
p_{x} \\
p_{y} \\
p_{z}
\end{array}\right),
$$

the angular momentum is $\mathbf{l}$ and $\mathbf{p}$ is the linear momentum. These 6 -dimensional vectors can be called co-screws as they are intended to be dual to the screws. These co-screws can also be used to represent wrenches, that is combinations of force and torque vectors,

$$
\mathcal{W}=\left(\begin{array}{l}
\boldsymbol{\tau} \\
\mathbf{F}
\end{array}\right) .
$$

It is very important to know how the group of rigid transformations $S E(3)$, acts on these vectors.

For screws, the action is given by the adjoint representation of the group on its Lie algebra. This can be written in terms of $6 \times 6$ matrices that have the partitioned form,

$$
H=\left(\begin{array}{cc}
R & 0 \\
T R & R
\end{array}\right),
$$

here $R$ is a $3 \times 3$ rotation matrix and $T$ is an anti-symmetric $3 \times 3$ matrix representing the translation vector $\mathbf{t}$. So that $T \mathbf{x}=\mathbf{t} \times \mathbf{x}$ for any vector $\mathbf{x}$.

Screws transform according to $\mathbf{s} \rightarrow H \mathbf{s}$ but co-screws transform under the dual representation; the co-adjoint representation, $\mathcal{P} \rightarrow H^{-T} \mathcal{P}$. Here $H^{-T}$ is short for $\left(H^{-1}\right)^{T}=\left(H^{T}\right)^{-1}$. The partitioned form of this matrix is,

$$
H^{-T}=\left(\begin{array}{cc}
R & T R \\
0 & R
\end{array}\right)
$$

The evaluation of a co-screw on a screw can be written as a matrix product,

$$
\mathcal{P}(\mathbf{s})=\mathcal{P}^{T} \mathbf{s} .
$$

This combination is clearly independent of the action of the rigid body motions; it is a scalar. This allows us to express the kinetic energy of the rigid body in terms of screws and co-screws,

$$
E_{K}=\frac{1}{2} \mathcal{P}(\mathbf{s})=\frac{1}{2}(\boldsymbol{\omega} \cdot \mathbf{l}+\mathbf{v} \cdot \mathbf{p})=\frac{1}{2}\left(\omega_{x} l_{x}+\omega_{y} l_{y}+\omega_{z} l_{z}+v_{x} p_{x}+v_{y} p_{y}+v_{z} p_{z}\right),
$$

where $\mathcal{P}$ is the momentum co-screw of the body and $\mathbf{s}$ is its velocity screw.

The inertia matrix of the body can be represented as a symmetric $6 \times 6$ matrix,

$$
N=\left(\begin{array}{cc}
I & m C \\
m C^{T} & m \mathrm{I}_{3}
\end{array}\right)
$$


Here $I$ is usual the $3 \times 3$ inertia matrix, while $I_{3}$ is the $3 \times 3$ identity matrix. The mass of the body is $m$ and $C$ is the anti-symmetric matrix representing the position vector of the centre of mass. The inertia maps velocities to momenta,

$$
\mathcal{P}=N \mathbf{s} .
$$

The transformation properties of the inertia matrix can be inferred from the fact that the kinetic energy must be invariant,

$$
N \longrightarrow H^{-T} N H^{-1} \text {. }
$$

Finally, we need the co-adjoint action of a screw on a co-screw, this gives the Coriolis terms in the equations of motion. The action will be denoted by curly brackets. The operation is dual to the adjoint action of the screws acting on themselves; the Lie bracket,

$$
\mathbf{s}_{1}^{T}\left\{\mathbf{s}_{2}, \mathcal{P}\right\}=\left[\mathbf{s}_{1}, \mathbf{s}_{2}\right]^{T} \mathcal{P} .
$$

In the partitioned form we have,

$$
\{\mathbf{s}, \mathcal{P}\}=\left\{\left(\begin{array}{l}
\boldsymbol{\omega} \\
\mathbf{v}
\end{array}\right),\left(\begin{array}{l}
\mathbf{l} \\
\mathbf{p}
\end{array}\right)\right\}=\left(\begin{array}{c}
\boldsymbol{\omega} \times \mathbf{l}+\mathbf{v} \times \mathbf{p} \\
\boldsymbol{\omega} \times \mathbf{p}
\end{array}\right) .
$$

The equation of motion for a single rigid body can now be written,

$$
N \frac{d}{d t} \mathbf{s}+\{\mathbf{s}, N \mathbf{s}\}=\mathcal{W},
$$

where $\mathcal{W}$ is the external wrench (force/torque co-screw) acting on the body.

This is not quite a simple combination of the Newton and Euler equations of motion. These are given in a fixed inertial frame, hence the inertia matrix changes as the body moves. As mentioned in the introduction, in robotics, it is convenient to use a single fixed inertial frame of reference since there will be several interacting rigid bodies.

\section{Rigid Transformations}

For kinematic problems it has been known for a long time that screws can be represented using the Clifford algebra $C \ell(0,3,1)$. This is the basis for Study's dual quaternions, see [11]. In the context of robot kinematics $C \ell(3,0,1)$ was used by Bayro-Corrochano and Kähle [3], however we will follow [14] here and base our considerations on $C \ell(0,3,1)$. Here, we have three generators which square to -1 and one which squares to 0 . Say, $e_{1}^{2}=e_{2}^{2}=e_{3}^{2}=-1$ and $e^{2}=0$.

In this algebra we can represent rotations by elements of the form,

$$
r=\cos \frac{\theta}{2}+\sin \frac{\theta}{2}\left(u_{x} e_{2} e_{3}+u_{y} e_{3} e_{1}+u_{z} e_{1} e_{2}\right)
$$

The angle of rotation is $\theta$ and $\left(u_{x}, u_{y}, u_{z}\right)^{T}$ is a unit vector in the direction of the rotation axis. This is simply the representation of rotations by quaternions, in a slightly different guise, just replace $e_{2} e_{3} \mapsto i, e_{3} e_{1} \mapsto j$ and $e_{1} e_{2} \mapsto k$. So, this is 
a double-valued representation, or rather a representation of the covering group $\operatorname{Spin}(3)$.

Translations are represented, in this algebra by elements of the form,

$$
t=1+\frac{1}{2}\left(t_{x} e_{1} e+t_{y} e_{2} e+t_{z} e_{3} e\right) .
$$

Rigid transformations are combinations of rotations and translations $g=t r$, and so will have the general form,

$$
g=\alpha_{0}+\alpha_{1} e_{2} e_{3}+\alpha_{2} e_{3} e_{1}+\alpha_{3} e_{1} e_{2}+\beta_{0} e e_{1} e_{2} e_{3}+\beta_{1} e_{1} e+\beta_{2} e_{2} e+\beta_{3} e_{3} e,
$$

where the $\alpha$ s and $\beta$ s are real coefficients. Notice that the term in $\beta_{0}$ has Clifford element $e e_{1} e_{2} e_{3}$ this could also have been written as $-\beta_{0} e_{1} e_{2} e_{3} e$, this choice is for consistency with dual quaternion approaches to the subject. The coefficients are not completely arbitrary, for such an element to be a rigid body motion it must satisfy the condition,

$$
g g^{*}=1 .
$$

Here the superscript $*$ denotes the Clifford conjugation, see [12, Chap. 9]. In terms of the coefficients the above equation gives just two relations,

$$
\alpha_{0}^{2}+\alpha_{1}^{2}+\alpha_{2}^{2}+\alpha_{3}^{2}=1 \quad \text { and } \quad \alpha_{0} \beta_{0}+\alpha_{1} \beta_{1}+\alpha_{2} \beta_{2}+\alpha_{3} \beta_{3}=0 .
$$

The Lie algebra of the group of rigid body motions can also be represented in this algebra. These are the screws, or more properly the twists, they are represented by arbitrary elements of grade 2 ,

$$
\mathbf{s}=\omega_{x} e_{2} e_{3}+\omega_{y} e_{3} e_{1}+\omega_{z} e_{1} e_{2}+v_{x} e_{1} e+v_{y} e_{2} e+v_{z} e_{3} e .
$$

As usual, $\left(\omega_{x}, \omega_{y}, \omega_{z}\right)^{T}$ is the angular velocity vector of the body and $\left(v_{x}, v_{y}, v_{z}\right)^{T}$ is the linear velocity vector of the body. The Lie bracket of a pair of screws $\mathbf{s}_{1}$ and $\mathbf{s}_{2}$ is simply their commutator,

$$
\left[\mathbf{s}_{1}, \mathbf{s}_{2}\right]=\frac{1}{2}\left(\mathbf{s}_{1} \mathbf{s}_{2}-\mathbf{s}_{2} \mathbf{s}_{1}\right) .
$$

The exponential map from the Lie algebra to the group is consistent with the Clifford algebra, given a Lie algebra element $\mathbf{z}$ we have,

$$
g=e^{\frac{1}{2} \mathbf{z}}=1+(\mathbf{z} / 2)+\frac{1}{2}(\mathbf{z} / 2)^{2}+\cdots,
$$

the factor $\frac{1}{2}$ here is due to the fact that we are working in the double cover of $S E(3)$.

The velocity screw comes from the time derivative of this relation. This is simple if the motion of the body is a uniform motion about a constant screw, $g=e^{\frac{t}{2} \mathbf{s}_{0}}$. It is easy to see that the derivative of this is,

$$
\frac{d}{d t} g=\frac{1}{2} \mathbf{s}_{0} g
$$


When the motion is more general, $g=e^{\frac{1}{2} \mathbf{z}(t)}$, then it is harder to show but nevertheless true that,

$$
\frac{d}{d t} g=\frac{1}{2} \mathbf{s} g
$$

where $\mathbf{s}$ is the velocity screw of the rigid body. The relationship between $\mathbf{s}$ and $\mathbf{z}$ is given by,

$$
\mathbf{s}=\sum_{i=0}^{\infty} \frac{1}{(i+1) !} \operatorname{ad}^{i}(\mathbf{z}) \frac{d}{d t} \mathbf{z},
$$

where $\operatorname{ad}^{i}(\mathbf{z}) \mathbf{y}=\left[\mathbf{z}, \operatorname{ad}^{i-1}(\mathbf{z}) \mathbf{y}\right]$ and $\operatorname{ad}^{1}(\mathbf{z}) \mathbf{y}=[\mathbf{z}, \mathbf{y}]$, see [4]

\section{The Shuffle Product}

The theory of exterior or Grassmann products in Clifford algebras is well established, see, [9, Chap. 3] for example. However we will also need the dual of this operation. In a non-degenerate Clifford algebra this could be done simply using the unit pseudo-scalar. That is the element $e_{12 \cdots n}=e_{1} e_{2} \cdots e_{n}$, with maximum grade. In [10] Lounesto gives the formula,

$$
b \vee c=\left(\left(b e_{12 \cdots n}^{-1}\right) \wedge\left(c e_{12 \cdots n}^{-1}\right)\right) e_{12 \cdots n}
$$

The algebra we are using is degenerate, $e_{12 \cdots n}^{-1}$ doesn't exist, so this shuffle operation has to be introduced explicitly.

The construction is borrowed directly from the theory of Grassmann-Cayley algebras, see [18] and [12, Chap. 10]. The shuffle product $\vee$, is defined as follows. Let $b=b_{1} \wedge b_{2} \wedge \cdots \wedge b_{j}$ and $c=c_{1} \wedge c_{2} \wedge \cdots \wedge c_{k}$ in a general Clifford algebra of dimension $n$, assuming $j+k \geq n$ we define

$$
b \vee c=\sum_{\sigma} \operatorname{sign}(\sigma) \operatorname{det}\left(b_{\sigma(1)}, \ldots, b_{\sigma(n-k)}, c_{1}, \ldots, c_{k}\right) b_{\sigma(n-k+1)} \wedge \cdots \wedge b_{\sigma(j)} .
$$

The sum is taken over all permutations $\sigma$ of $1,2, \ldots, j$ such that $\sigma(1)<\sigma(2)<$ $\cdots<\sigma(n-k)$ and $\sigma(n-k+1)<\sigma(n-k+2)<\cdots<\sigma(j)$.

Each $b_{i}$ can be written as a sum of basis elements,

$$
b_{i}=b_{i 1} e_{1}+b_{i 2} e_{2}+\cdots+b_{i n} e_{n} .
$$

The determinant in the above definition is the determinant of the matrix whose columns are the coefficients $b_{\sigma(1) i}, b_{\sigma(2) i}, \ldots, c_{\sigma(k) i}$. The shuffle product is then extended to the entire Clifford algebra by demanding that it distributes over addition. When $j+k<n$ the shuffle product is zero.

For example, consider $e_{1} e_{2} \vee e_{3} e$ in $C \ell(0,3,1)$. For an orthogonal basis we can confuse the Clifford product of basis elements with the exterior product, so,

$$
e_{1} e_{2} \vee e_{3} e=\operatorname{det}\left(e_{1}, e_{2}, e_{3}, e\right)=1 \text {, }
$$

where $e$ has been taken to be the fourth basis element " $e_{4}$ ". 
As another example, consider the algebra $C \ell(0,6,2)$ with the orthogonal set of generators, $a_{1}, a_{2}, a_{3}, a, e_{1}, e_{2}, e_{3}, e$ with that order. Suppose we need to compute $a_{1} a_{2} a_{3} a \vee a_{3} a_{1} e_{1} e_{2} e_{3} e$,

$$
\begin{aligned}
& a_{1} a_{2} a_{3} a \vee a_{3} a_{1} e_{1} e_{2} e_{3} e=\operatorname{det}\left(a_{1} a_{2} a_{3} a_{1} e_{1} e_{2} e_{3} e\right) a_{3} a+\cdots \\
& -\operatorname{det}\left(a_{2} a a_{3} a_{1} e_{1} e_{2} e_{3} e\right) a_{1} a_{3}+\cdots+\operatorname{det}\left(a_{3} a a_{3} a_{1} e_{1} e_{2} e_{3} e\right) a_{1} a_{2} .
\end{aligned}
$$

Clearly only the middle term on the right-hand-side is non-zero so we have the result,

$$
a_{1} a_{2} a_{3} a \vee a_{3} a_{1} e_{1} e_{2} e_{3} e=a_{3} a_{1}
$$

\section{Momenta and Inertia}

In order to include momenta and inertias we 'double' the algebra and use the Clifford algebra $C \ell(0,6,2)$. We will label the generators, $e_{1}, e_{2}, e_{3}, e$ and $a_{1}, a_{2}, a_{3}, a$, and assume $a_{i}^{2}=e_{j}^{2}=-1$ and $a^{2}=e^{2}=0$.

In this algebra we can represent the co-screws with elements of grade 2 of the form,

$$
\mathcal{P}=p_{x} a_{2} a_{3}+p_{y} a_{3} a_{1}+p_{z} a_{1} a_{2}+l_{x} a_{1} a+l_{y} a_{2} a+l_{z} a_{3} a .
$$

It is easy to see that the action of the group of rigid body motions on these elements is exactly the same as the action on the screws but using as instead of es.

So let us introduce a new operation, an involution which exchanges as for es. If we write this with an over bar then $\bar{a}_{i}=e_{i}$ and $\bar{e}_{i}=a_{i}$.

Now the group action on momenta can be written,

$$
\mathcal{P} \longrightarrow \bar{g} \mathcal{P} \bar{g}^{*},
$$

where $g$ would be the corresponding group element that acts on the screws.

The evaluation map of a co-screw on a screw can be written using the invariant element $Q_{0}$,

$$
Q_{0}=a_{2} a_{3} e_{1} e+a_{3} a_{1} e_{2} e+a_{1} a_{2} e_{3} e+a_{1} a e_{2} e_{3}+a_{2} a e_{3} e_{1}+a_{3} a e_{1} e_{2} .
$$

Now the evaluation, can have either of the two forms,

$$
\mathcal{P}(\mathbf{s})=\mathcal{P} \vee\left(Q_{0} \wedge \mathbf{s}\right)=\left(\mathcal{P} \wedge Q_{0}\right) \vee \mathbf{s} .
$$

The invariance of the element $Q_{0}$ here is with respect to the group action, $(g \bar{g}) Q_{0}(g \bar{g})^{*}$. That is, $Q_{0}=(g \bar{g}) Q_{0}(g \bar{g})^{*}$, however, this element is not invariant under the action of either $g$ or $\bar{g}$ alone, $Q_{0} \neq g Q_{0} g^{*}$ and $Q_{0} \neq \bar{g} Q_{0} \bar{g}^{*}$. Notice that the evaluation here is very similar to the reciprocal product in traditional Screw theory.

Explicitly, with $\mathbf{s}$ as in section 3 we have,

$$
Q_{0} \wedge \mathbf{s}=\left(\omega_{x} a_{2} a_{3}+\omega_{y} a_{3} a_{1}+\omega_{z} a_{1} a_{2}+v_{x} a_{1} a+v_{y} a_{2} a+v_{z} a_{3} a\right) e_{1} e_{2} e_{3} e,
$$

since the exterior product is the same as the Clifford product on an orthogonal basis - except that terms containing a basis element twice vanish. Taking the shuffle of this with $\mathcal{P}$ we get,

$$
\mathcal{P} \vee\left(Q_{0} \wedge \mathbf{s}\right)=\omega_{x} l_{x}+\omega_{y} l_{y}+\omega_{z} l_{z}+v_{x} p_{x}+v_{y} p_{y}+v_{z} p_{z}
$$


This time the cross terms vanish because the determinant in the shuffle product will contain repeated columns.

Next we look at the inertia matrix. Notice that since any inertia matrix can be transformed to a diagonal matrix by a suitable rigid body motion, if we get the group action on inertias correct then we only have to consider diagonal inertia matrices. That is, if the equations work for diagonal inertia matrices they will work for general inertia matrices. This saves a considerable amount of work verifying our constructions. Now a diagonal inertia matrix can be represented as,

$$
N=d_{x} a_{1} a e_{1} e+d_{y} a_{2} a e_{2} e+d_{z} a_{3} a e_{3} e+m a_{2} a_{3} e_{2} e_{3}+m a_{3} a_{1} e_{3} e_{1}+m a_{1} a_{2} e_{1} e_{2},
$$

where as usual $m$ represents the mass of the body, and $d_{i}$ is the mass times the square of the radius of gyration about the principle axis $i$.

To construct the map from velocities to momenta we need another invariant, $A=a_{1} a_{2} a_{3} a$. This element clearly commutes with any $g$ so unlike the previous invariant $A=g A g^{*}$. It is also easy to see that $A=\bar{g} A \bar{g}^{*}$ so clearly $A=(g \bar{g}) A(g \bar{g})^{*}$. Now the momentum of the rigid body is given by,

$$
\mathcal{P}=A \vee(N \wedge \mathbf{s}) \text {. }
$$

Explicitly, with the diagonal inertia as above and $s$ as in section 3, we get,

$$
\mathcal{P}=m v_{x} a_{2} a_{3}+m v_{y} a_{3} a_{1}+m v_{z} a_{1} a_{2}+d_{x} \omega_{x} a_{1} a+d_{y} \omega_{y} a_{2} a+d_{z} \omega_{z} a_{3} a,
$$

as would be expected from elementary mechanics.

Next we introduce two small results, firstly we have that: $h(a \wedge b) h^{*}=\left(h a h^{*}\right) \wedge$ $\left(h b h^{*}\right)$, for any elements of the Clifford algebra $a, b$ and $h$ an element which satisfies $h h^{*}=1$ and $h \mathbf{x} h^{*}$ is a vector for any vector $\mathbf{x}$. For any two vectors $\mathbf{x}$ and $\mathbf{y}$ we have,

$$
h(\mathbf{x} \wedge \mathbf{y}) h^{*}=h \frac{1}{2}(\mathbf{x y}-\mathbf{y} \mathbf{x}) h^{*}=\frac{1}{2}\left(h \mathbf{x} h^{*} h \mathbf{y} h^{*}-h \mathbf{y} h^{*} h \mathbf{x} h^{*}\right)=\left(h \mathbf{x} h^{*}\right) \wedge\left(h \mathbf{y} h^{*}\right) .
$$

Just as the definition of the exterior product can be extended by linearity and associativity to the whole algebra, so can this result since conjugation by $h$ is a linear operation.

The second result is really the dual of the first, that is: $h(a \vee b) h^{*}=\left(h a h^{*}\right) \vee$ $\left(h b h^{*}\right)$, for any elements of the Clifford algebra $a, b$ and $h$ as above. Note however that the shuffle product will be zero unless the sum of the grades of the lowest grade terms in $a$ and $b$ is more than or equal to the dimension of the Clifford algebra.

In a non-degenerate Clifford algebra this result would not require any extra proof because of the definition of the shuffle product using the pseudo-scalar as in section 4 above. For degenerate Clifford algebras, such as the ones considered in this work this result can be shown quite simply. First observe that for vectors $\mathbf{x}_{1}, \mathbf{x}_{2}, \cdots \mathbf{x}_{n}$ we have,

$$
\operatorname{det}\left(\left(h \mathbf{x}_{1} h^{*}\right),\left(h \mathbf{x}_{2} h^{*}\right), \cdots\left(h \mathbf{x}_{n} h^{*}\right)\right)=\operatorname{det}\left(\mathbf{x}_{1}, \mathbf{x}_{2}, \cdots \mathbf{x}_{n}\right) .
$$


This can be seen by considering the action of $h$ and $h^{*}$ on vectors as being represented by matrix multiplication. The $n \times n$ matrices representing $h$ and $h^{*}$ will have determinants which are mutually reciprocal since $h h^{*}=1$. Combining this with the first result and the definition of the shuffle product given in section 4 above yields the second result. Finally here, we observe that $g \bar{g}$ clearly satisfies the conditions $(g \bar{g})(g \bar{g})^{*}=1$ and $(g \bar{g}) \mathbf{x}(g \bar{g})^{*}$ is a vector for all vectors $\mathbf{x}$.

Now to find the action of $S E(3)$ on the inertia we use the two results above. Transforming the equation for the momentum co-screw using conjugation by $g \bar{g}$ gives,

$$
(g \bar{g}) \mathcal{P}(g \bar{g})^{*}=(g \bar{g})(A \vee(N \wedge \mathbf{s}))(g \bar{g})^{*}=(g \bar{g}) A(g \bar{g})^{*} \vee\left((g \bar{g}) N(g \bar{g})^{*} \wedge(g \bar{g}) \mathbf{s}(g \bar{g})^{*}\right)
$$

using the results above. Since $A$ is invariant, $\mathcal{P}$ commutes with $g$ and s commutes with $\bar{g}$ we have,

$$
\bar{g} \mathcal{P} \bar{g}^{*}=A \vee\left(g \bar{g} N \bar{g}^{*} g^{*} \wedge g \mathbf{s} g^{*}\right) .
$$

Hence we can deduce that the inertia must transform according to,

$$
N \longrightarrow(g \bar{g}) N(g \bar{g})^{*} .
$$

Notice that in fact the same transformation law could be used for, the screws and co-screw, $\mathbf{s} \rightarrow(g \bar{g}) \mathbf{s}(g \bar{g})^{*}$ and $\mathcal{P} \rightarrow(g \bar{g}) \mathcal{P}(g \bar{g})^{*}$.

As an example, consider how the diagonal inertia above transforms under a translation of $t_{x}$ in the $x$-direction. For this transformation we have,

$$
g=1+\frac{1}{2} t_{x} e_{1} e
$$

and hence,

$$
g \bar{g}=\left(1+\frac{1}{2} t_{x} e_{1} e\right)\left(1+\frac{1}{2} t_{x} a_{1} a\right)=1+\frac{1}{2} t_{x} a_{1} a+\frac{1}{2} t_{x} e_{1} e+\frac{1}{4} t_{x}^{2} a_{1} a e_{1} e .
$$

For hand calculation it is probably better to perform the multiplications by $g$ and $\bar{g}$ separately. The result is,

$$
\begin{gathered}
g \bar{g} N \bar{g}^{*} g^{*}=\quad d_{x} a_{1} a e_{1} e+\left(d_{y}+m t_{x}^{2}\right) a_{2} a e_{2} e+\left(d_{z}+m t_{x}^{2}\right) a_{3} a e_{3} e+ \\
m t_{x} a_{3} a e_{3} e_{1}-m t_{x} a_{2} a e_{1} e_{2}+ \\
m t_{x} a_{3} a_{1} e_{3} e-m t_{x} a_{1} a_{2} e_{2} e+ \\
m a_{2} a_{3} e_{2} e_{3}+m a_{3} a_{1} e_{3} e_{1}+m a_{1} a_{2} e_{1} e_{2} .
\end{gathered}
$$

Compare this with the corresponding $6 \times 6$ inertia matrix,

$$
\left(\begin{array}{cccccc}
d_{x} & 0 & 0 & 0 & 0 & 0 \\
0 & d_{y}+m t_{x}^{2} & 0 & 0 & 0 & -m t_{x} \\
0 & 0 & d_{z}+m t_{x}^{2} & 0 & m t_{x} & 0 \\
0 & 0 & 0 & m & 0 & 0 \\
0 & 0 & m t_{x} & 0 & m & 0 \\
0 & -m t_{x} & 0 & 0 & 0 & m
\end{array}\right) .
$$


Finally here, the kinetic energy of the rigid body can be written,

$$
E_{k}=\frac{1}{2} \mathcal{P} \vee\left(Q_{0} \wedge \mathbf{s}\right)=\frac{1}{2} A \vee\left(Q_{0} \wedge \mathbf{s}\right) \vee(N \wedge \mathbf{s})
$$

Notice that the combination $A \vee\left(Q_{0} \wedge \mathbf{s}\right)$ produces,

$$
A \vee\left(Q_{0} \wedge \mathbf{s}\right)=\omega_{x} a_{2} a_{3}+\omega_{y} a_{3} a_{1}+\omega_{z} a_{1} a_{2}+v_{x} a_{1} a+v_{y} a_{2} a+v_{z} a_{3} a
$$

This, of course is the same as $\overline{\mathbf{s}}$. So the kinetic energy can be written neatly as,

$$
E_{k}=\frac{1}{2} \overline{\mathbf{s}} \vee(N \wedge \mathbf{s})
$$

\section{Equations of Motion}

We need one final operation before we can write down the equations of motion for a rigid body; the co-adjoint action. This can be found from the group action on the co-screws given in the previous section. Let us suppose that a co-screw is subject to a uniform motion about a screw. The relevant group elements can be written as exponentials, $g=e^{\frac{t}{2} \mathbf{s}}$ where $t$ is time. Hence, as a function of time the co-screw is given by,

$$
\mathcal{P}(t)=e^{\frac{t}{2} \overline{\mathbf{s}}} \mathcal{P} e^{-\frac{t}{2} \overline{\mathbf{s}}} .
$$

Now if we differentiate this with respect to $t$ and then set $t=0$, we get the co-adjoint action of the screw $\mathbf{s}$ on the co-screw $\mathcal{P}$,

$$
\{\mathbf{s}, \mathcal{P}\}=\frac{1}{2}(\overline{\mathbf{s}} \mathcal{P}-\mathcal{P} \overline{\mathbf{s}}) .
$$

Computation shows that this agrees with the formula given in section 2 .

The equations of motion can be derived very simply now. According to Newton's second law, the force applied to the body is equal to the rate of change of momentum, in our notation this becomes,

$$
\frac{d}{d t} \mathcal{P}=\mathcal{W}
$$

where $\mathcal{W}$ is the applied wrench. The momentum is given by, $\mathcal{P}=A \vee(N \wedge \mathbf{s})$, as we saw above. The derivatives of the factors here are simple to find, $A$ is an invariant so its derivative is zero, the screw $\mathbf{s}$ is the unknown here so we simply write $\frac{d}{d t} \mathbf{s}=\dot{\mathbf{s}}$. The derivative of the inertia matrix can be found by differentiating the group action,

$$
\frac{d}{d t} N=\frac{1}{2}(\mathbf{s} N-N \mathbf{s})+\frac{1}{2}(\overline{\mathbf{s}} N-N \overline{\mathbf{s}}) .
$$

Now $(\mathbf{s} N-N \mathbf{s}) \wedge \mathbf{s}=0$ so when we combine these results to form the derivative of the momenta we get,

$$
\frac{d}{d t} \mathcal{P}=A \vee(N \wedge \dot{\mathbf{s}})+\frac{1}{2} A \vee((\overline{\mathbf{s}} N-N \overline{\mathbf{s}}) \wedge \mathbf{s})
$$


This agrees with the results given in section 2 , since the second term on the righthand side here is,

$$
\frac{1}{2}(\overline{\mathbf{s}}(A \vee(N \wedge \mathbf{s}))-(A \vee(N \wedge \mathbf{s})) \overline{\mathbf{s}})=\{\mathbf{s}, A \vee(N \wedge \mathbf{s})\}=\{\mathbf{s}, \mathcal{P}\}
$$

It should also be possible to derive this equation from the expression for the kinetic energy using Lagrangian mechanics.

Notice that we can tidy our Clifford equations of motion a little by introducing the invariant element $E=\bar{A}=e_{1} e_{2} e_{3} e$. Clearly, we have that $A E \vee c=c$ for any element $c$ in the algebra, so multiplying our equation by $E$ gives,

$$
N \wedge \dot{\mathbf{s}}+\frac{1}{2}((\overline{\mathbf{s}} N-N \overline{\mathbf{s}}) \wedge \mathbf{s})=\mathcal{W} E .
$$

\section{Conclusions}

As mentioned in the introduction, one of the main motivations for this work was to cast the dynamics of robots into a Clifford algebra form. This can now be done quite simply, at least for serial robot arms, we can just imitate the analysis given in [12] for example, and in fact this has been done in [13].

A more significant achievement of this work is that we have been able to find a Clifford algebra which contains the symmetric tensor square of the adjoint representation of the group of rigid body motions $S E(3)$. Clifford algebras are more usually associated with the anti-symmetric representations. The methods used here have some similarities to the work of Sobczyk [15], where general linear algebra is represented using Clifford algebra.

Actually, inertia matrices lie in a subspace of the symmetric square of the adjoint representation. However, it is clear that the Clifford algebra $C \ell(0,6,2)$ contains all of this representation. This means that it would be relatively easy to include first order elasticity and damping into this presentation of mechanics. This is because the damping matrix and stiffness matrix of such systems are also symmetric $6 \times 6$ matrices with the same transformation properties as the inertia matrix.

Notice that this means that inertias are honest elements of the Clifford algebra, grade 4 elements in fact. Moreover these inertias correspond to the $6 \times 6$ inertia matrices of screw theory. This means that we don't need to introduce the parallel axis theorem for inertias, it is contained in, and generalised by, the transformation properties of the inertia elements.

Of course this is the main advantage of using Clifford algebras in general, everything is in the algebra. Not just the inertias, velocities and momenta but also the elements of the group of rigid body motions. All the relations between physical quantities are given by standard operations in the algebra. This includes the Lie bracket of screws and the adjoint action of screws on co-screws. So to perform computations all we need to know is how to do computations in any Clifford algebra. 
The algebra we have used $C \ell(0,6,2)$ is rather large, a key question is, can we find a smaller algebra in which to represent the dynamics of rigid bodies? Certainly everything we have done uses even graded elements of the algebra, hence by standard theory of Clifford algebra all our constructions could be reduced to $C \ell(0,5,2)$. Then again if size was the only consideration we could resort to Hestenes' original $C \ell(3)$ formulation. The question is really: what is the smallest Clifford algebra that contains a copy of the symmetric square of the adjoint representation of $S E(3)$ ?

\section{References}

[1] E.T. Abou El Dahab, 2000. "A Formulation of Hamiltonian Mechanics using Geometric Algebra". Advances in Applied Clifford Algebras, 10(2):217-223.

[2] L. Dorst, D. Fontijne, S. Mann, 2007 Geometric Algebra for Computer Science: An Object-Oriented approach to Geometry, Morgan Kaufmann, San Francisco.

[3] E. Bayro-Corrochano and D. Kähle, 2000. "Motor Algebra Approach for Computing the Kinematics of Robot Manipulators". Journal of Robotic Systems, 17(9):495-516.

[4] F. Hausdorff, 1906. "Die Symbolische Exponential Formel in den Grupen Theorie". Berichte de Sächichen Akademie de Wissenschaften (Math Phys Klasse) 58:19-48.

[5] D. Hestenes, 1999. New Foundations for Classical Mechanics 2nd ed. D. Reidel, Dordrecht.

[6] D. Hestenes, 2001. "Old Wine in New Bottles: A New Algebraic Framework for Computational Geometry", chapter 1, pp. 3-17. in Applied Clifford Algebra in Cybernetics, Robotics, Image Processing and Engineering, eds. E. Bayro-Corrochano and G. Sobczyk. Birkhäuser, Boston.

[7] D. Hestenes and E. Fasse, 2002 "Homogeneous Rigid Body Mechanics with Elastic Coupling". in Applications of geometric algebra in computer science and engineering, eds. L.Dorst, C. Doran and J. Lasenby. Birkhäuser, Boston, pp. 197-212.

[8] I. Millington, 2007 Game Physics Engine Development, Morgan Kaufmann, San Francisco.

[9] P. Lounesto, 2001. Clifford Algebras and Spinors, 2nd edition. LMS Lecture Note Series 286, Cambridge University Press, Cambridge.

[10] P. Lounesto, 2004. "Introduction to Clifford Algebras", Lecture 1, pp. 1-29, in Lectures on Clifford (geometric) algebras and applications. eds. R. Abłamowicz and G. Sobczyk, Birkhäuser, Boston.

[11] I.R. Porteous, 1981. Topological Geometry. Cambridge University Press, Cambridge, second edition.

[12] J.M. Selig, 2005. Geometric Fundamentals of Robotics. Springer Verlag, New York.

[13] J.M. Selig, 2005. "Clifford Algebra and Robot Dynamics", Chapter 19, pp. 637-658, in Handbook of Computational geometry for Pattern recognition, Vision, Neurocomputing and Robotics, editor E. Bayro-Corrochano, Springer Verlag, New York.

[14] J.M. Selig, 2001. "Robot Kinematics and Flags". chapter 11, pp. 211-234, in Applied Clifford Algebra in Cybernetics, Robotics, Image Processing and Engineering, eds. E. Bayro-Corrochano and G. Sobczyk. Birkhäuser, Boston. 
[15] G. Sobczyk, 2001. "Universal Geometric Algebra". chapter 2, pp. 18-41, in Applied Clifford Algebra in Cybernetics, Robotics, Image Processing and Engineering, eds. E. Bayro-Corrochano and G. Sobczyk. Birkhäuser, Boston.

[16] J.C. Trinkle, J.S. Pang, S. Sudarsky and G. Lo, 1997. "On Dynamic Multi-rigid-body Contact Problems with Coulomb friction". Zeitschrift für Angewandte Matematik und Mechanik, 77(4):267-279.

[17] J. M. Van Verth, L. M. Bishop, 2004. Essential Mathematics for Games and Interactive Applications: A Programmers Guide, Morgan Kaufmann, San Francisco.

[18] N. White, 1994. "Grassmann-Cayley Algebra and Robotics". J. Intell. Robot Syst., 11:97-107.

J.M. Selig

Faculty of Business, Computing and Information Management

London South Bank University,

London SE1 0AA,

UK.

e-mail: seligjm@lsbu.ac.uk

E. Bayro-Corrochano

Centro de Investigacion y de Estudios Avanzados,

Guadalajara,

Jalisco 44550,

Mexico.

e-mail: edb@gdl. cinvestav .mx 